\title{
Proceso de valuación de viviendas unifamiliares como medio de control en Orizaba, Veracruz, México
}

\section{Single-family home valuation process as a means of control in Orizaba, Veracruz, Mexico}

\author{
Héctor Isaac Merino Xotlanihua ${ }^{1}$, Francisco José Martín del Campo Saray ${ }^{2}$ \\ ${ }^{1}$ Maestría en Valuación; Colaborador de la Universidad de Guadalajara, México; hecmer 20@hotmail.com; \\ https://orcid.org/: 0000-0003-1557-3074 \\ ${ }^{2}$ Doctorado en Arquitectura y Maestría en Valuación; Profesor de la Universidad de Guadalajara, México; \\ fsaray7@hotmail.com; https://orcid.org/: 00-0001-7211-5366
}

Resumen: Se aprecia que el patrón de encarecimiento de inmuebles en muchas ciudades y la valuación de viviendas es un tema de interés. Esto debido a factores que crean y modifican su valor: físicos, económicos, sociales y políticos. También influyen la escasez del suelo para construir, incremento de la población que demanda la adquisición de vivienda, establecimiento de negocios y generación de fuentes de empleo que denotan liquidez en una sociedad. El siguiente trabajo tuvo como objetivo categorizar la valuación de viviendas unifamiliares como medio de control en Orizaba, Veracruz, México, el método de valuación propuesto denominado "método de comercialización", retoma procedimientos del enfoque de costos, método del cual permitió encontrar un factor de calidad que fuera aplicado a los bienes inmuebles de manera justa y no basado en intereses de utilidades empresariales y favoreciendo a los habitantes que utilizan un crédito hipotecario y/o de vivienda.

Palabras clave: valuación inmobiliaria, vivienda unifamiliar, método de comercialización.

\begin{abstract}
It is appreciated that the pattern of real estate prices is observed in many cities and the valuation of homes is a topic of interest. This is due to factors that create and modify its value: physical, economic, social and political. The scarcity of land to build, an increase in the population that demands the acquisition of housing, the establishment of businesses and the generation of sources of employment that denote liquidity in a society also influence. The objective of this work was to categorize the valuation of single-family homes as a means of control in Orizaba, Veracruz, Mexico, the proposed valuation method called "marketing method", takes up procedures of the cost approach, a method which allowed finding a factor of quality that was applied to real estate in a fair way and not based on business profit interests and favoring the inhabitants who use a mortgage and / or housing loan.
\end{abstract}

Key words: real estate valuation, single-family home, marketing method. 


\section{Introducción}

La valuación de viviendas unifamiliares nuevas que pueden ser adquiridas por créditos hipotecarios como Fovissste, Infonavit o algún cofinanciamiento con apoyo de los bancos, se vuelve una actividad clave, para que las personas puedan adquirir una propiedad a un precio justo. Esta situación resulta cada vez más difícil para la clase trabajadora, al ser créditos que deben pagar varios años; en los que, si se compra una casa a un precio muy elevado, provoca una mensualidad alta que no pueden solventar, porque su salario no les brinda la oportunidad de cubrir sus necesidades básicas, proveer los gastos fijos que se tienen y depositar la letra de una casa. Por esta razón, la valuación es crucial para no emitir precios por encima de su valor estimado.

Por consiguiente, comparar precios de ciudades diversas con condiciones similares a la ciudad de Orizaba, Veracruz, México, fueron fundamentales para utilizar este trabajo como una medida de control, de análisis y desarrollo, que puede aplicarse antes de tasar un inmueble y determinar precios que sólo convengan al sector inmobiliario.

El alza del valor del suelo, de los impuestos como el predial y los servicios que cobran los municipios, son parte de la repercusión que puede suscitar un alza de precios en las viviendas de manera no justificada y acelerada. La depreciación del salario mínimo, también se ve afectada ante el valor de los objetos cuando se encarecen dichos inmuebles.

Se puede decir, que una ciudad exitosa en servicios, infraestructura, equipamiento y mobiliario urbano, espacios recreativos y de esparcimiento, historia y arte, tiende a encarecerse; sin embargo, esto no es justificación para que se tasen las viviendas de manera irresponsable sin hacer un avalúo detallado que de fe del valor que se emite. Este trabajo no pretende derrocar el mercado inmobiliario ni cerrar empresas de este tipo, pero sí, el de no caer en actos de corrupción que favorezcan a particulares y empresarios que, a su vez, afecta a la economía de una población.

La ciudad de Orizaba, Veracruz, México, se encuentra ubicada en la zona centro del estado de Veracruz en la región de las montañas altas, entre los paralelos $18^{\circ} 50^{\prime}$ y $18^{\circ} 53^{\prime}$ de latitud norte; los meridianos $97^{\circ} 04^{\prime}$ y $97^{\circ} 10^{\prime}$ de longitud oeste; con una temperatura media anual de $23^{\circ} \mathrm{C}$, máxima promedio de $32^{\circ} \mathrm{C}$ y mínima promedio de $13^{\circ} \mathrm{C}$, precipitación 
media pluvial de $1500 \mathrm{~mm}$ anuales, humedad relativa promedio del $80 \%$ y una altitud de $1230 \mathrm{~m}$ sobre el nivel del mar. Colinda con los municipios de: Ixhuatlancillo, Mariano Escobedo, Atzacan, Ixtaczoquitlán, Huiloapan, Rafael Delgado y Río Blanco (Cuadernilos municipales, 2016). Ver figura 1.

\section{Figura 1. Localización de Orizaba, Veracruz, México.}

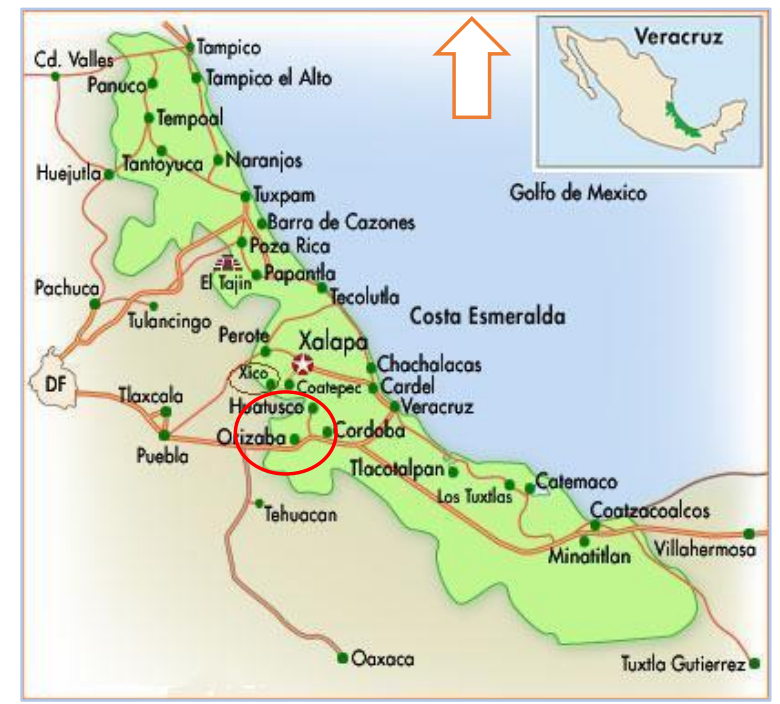

Fuente: Obtenido de Google.com

Se menciona que, del año 2015 al 2019 las viviendas en Orizaba, Veracruz, tuvieron precios elevados en relación con otras ciudades de mayor tamaño e importancia de la entidad como Xalapa, el Puerto de Veracruz, Boca del Río; y ciudades de otro estado como lo fue: Puebla y San Andrés Cholula, que sobrepasan el valor del suelo más los costos de producción, indirectos y utilidades, que genera el construir una casa habitación. Así lo indicó el periódico El Mundo en su artículo de la portada del 28 de octubre de 2018, titulado: "Se dispara plusvalía en Orizaba", en donde se menciona que "Mientras en el puerto de Veracruz se pueden ubicar casas nuevas, de hasta $1^{\prime} 500,000$ pesos mexicanos $(\$ 75,037$ dólares americanos), en Orizaba, casas con características similares, incluso en los límites del municipio alcanzan los 2’200,000 pesos mexicanos (\$110,055 dólares americanos)". (Carrillo, 2018, p.1)

El mercado inmobiliario se ha elevado en demasía y se han apoyado en factores como los atractivos turísticos de la ciudad, la denominación de pueblo mágico, la construcción de 
plazas comerciales, la recuperación y construcción de espacios públicos de recreación y esparcimiento; además, de la llegada de negocios y marcas comerciales a la ciudad. Estos son factores que, sin lugar a duda, mejoran los servicios, el movimiento económico y la calidad de vida de sus habitantes $y$, por consiguiente, el valor del suelo y de sus construcciones.

"La ciudad de Orizaba es considerado el pueblo mágico favorito de los viajeros" Ochoa (2017), según la revista México desconocido.com, en encuesta que se realizó del 10 al 14 de noviembre del 2017, se considera una de las mejores ciudades del estado de Veracruz para vivir y con mayores atractivos turísticos, además de, ofrecer eventos culturales del gusto de sus habitantes.

De 2014 a 2019 las inmobiliarias y empresas desarrolladoras de viviendas, han encarecido el mercado, significativamente, lo que convierte a Orizaba en una ciudad costosa, sin haber tomado en cuenta: la zona o colonia donde se ubican los inmuebles, el contexto, el tipo de vivienda que predomina, el equipamiento urbano y los servicios del lugar para determinar un valor justo de las viviendas; sino todo lo contrario, a mayor plusvalía sólo se incrementa el precio de venta sin hacer un análisis de lo antes mencionado, como si lo único que interesara fuera obtener utilidades establecidas por ellas mismas, sin perder ningún porcentaje de las ventas y no perder valor a ninguna de sus inversiones.

Es importante resaltar que el mismo diseño de vivienda con los mismos acabados, no tiene, forzosamente, que tener el mismo valor cuando están construidos en diferentes lugares; esto debido a sus factores exógenos, estatus socioeconómico del lugar, actividad económica de la zona y plusvalía que lo rodea. Sin embargo, existen viviendas y desarrollos en donde se omiten estos factores y son tasados al mismo precio todos los inmuebles.

Este proyecto mejora los conocimientos para analizar el mercado inmobiliario, para evaluar los precios de venta; y brinda un número mayor de elementos al soporte técnico de los dictámenes valuatorios que complementa los métodos establecidos para valuar una vivienda, toma aspectos como análisis de los Índices Nacionales de Precios al Consumidor (INPC) publicados por SHCP (2018), que son aspectos que miden la inflación en el país, con 
los cuales, se analiza una vivienda comprada hace diez años donde se actualiza su valor al año 2019 para realizar comparativas con el mercado inmobiliario.

Se piensa que, el mayor beneficio que presenta este estudio es establecer medidas de control para no aumentar los precios de las viviendas en la ciudad de Orizaba, Veracruz, de manera injustificada y evitar incrementos en el mercado inmobiliario que no sean producto de la inflación que se presenta en México.

A continuación, se presentan teorías de autores que brindaron sustento a la investigación;

Para hablar de valuación primero se define el concepto de valor, el cual representa el precio más probable que compradores y vendedores establecerán para un bien o servicio que está disponible para su compra. El valor establece un precio hipotético o teórico. De modo que no es un hecho, sino una estimación del precio más probable de un bien o servicio en un momento determinado (López, Aznar, González y Guijarro 2012 p. 3)

En consecuencia, y en función de la definición anterior, se establece que valorar como acción es asociar una cantidad de dinero a un específico bien o derecho, en función de sus condiciones y, como consecuencia, de un escenario de mercado (González, Turmo y Villaronga, 2006).

Para el ingeniero Roberto Loria (2012) en el prólogo del libro “Valoración inmobiliaria, Métodos y Aplicaciones", el cual, redactó para la presentación del mismo; menciona que "La valuación no es un campo estático sino dinámico y máxime al no ser una ciencia exacta, cada día nos invita a investigar, polemizar, a no ser dogmáticos, cuestionar métodos y a estudiar" (López, Aznar, González y Guijarro 2012 p. 8).

Por otro lado, Moctezuma y Veyna (2006) señalan que el conocimiento del mercado era la forma de instituir las ventajas entre valuadores, esta disponibilidad y manejo de datos, hacen la diferencia por el efecto de no disponer de cantidad suficiente de comparables, que complica el proceso de estimación masiva cuando se trata de valorar propiedades comerciales y no, simplemente, residenciales. Este tipo de valoraciones 
masivas causan discusión pues no dejan de exponer que es importante la percepción y conocimiento del valuador.

Por otra parte, en las Normas Internacionales de Valoración Meca et al, (2009), se define a la valoración como "el proceso de estimar un valor".

De acuerdo con el INDAABIN en su glosario de términos en materia de avalúos que emite la Dirección General de Avalúos y Obras, un avalúo es: El resultado del proceso de estimar el valor de un bien que determina la medida de su poder de cambio en unidades monetarias y a una fecha específica, definiéndolo también como, un dictamen técnico en del valor de un bien a partir de sus condiciones físicas, su uso, ubicación y estudio de mercado (INDAABIN, Marco normativo en materia valuatoria, 2019).

De acuerdo con información de bibliografía especializada, se enumeraron tendencias de consumo en México que se debieron considerar, si se quería mejorar en las ventas. A continuación, se mencionan tres de ellas:

1. Los consumidores aprecian más los productos nuevos e innovadores:

- $72 \%$ de los mexicanos prefiere productos nuevos sobre las marcas que consume.

- 6 de cada 10 consumidores realizan búsquedas de beneficios antes de comprar.

- $77 \%$ declaró que pagaría más por un producto nuevo, si este tiene mejor calidad.

2. El ambiente social es uno de los motores para la prueba de nuevos productos:

- $86 \%$ de los consumidores dijo que probaría las marcas en la cuales los demás confían.

- 8 de cada 10 confía en las recomendaciones de otros consumidores.

- $61 \%$ de los compradores confían en sellos de calidad y premios obtenidos por los productos.

3. Los consumidores mexicanos buscan el mejor precio sin renunciar a la calidad:

- 7 de cada 10 comentan que su prioridad es la calidad antes que la cantidad.

- El $90 \%$ se siente orgulloso si le dan un valor agregado por su dinero.

- Sólo el 70\% compra marcas genéricas, a comparación de países como Estados Unidos y Canadá donde $90 \%$ las consumen.

Como se puede observar los consumidores están ponderando la calidad de los productos por encima de la cantidad, pero buscan el mejor precio acorde a sus posibilidades 
económicas. La recomendación de otros usuarios y la certificación o sellos de calidad de las mercancías son de vital importancia y tomadas en cuenta al momento de comprarlas. Los consumidores se están alejando cada vez más de los productos fabricados en serie y optan por mercancías de mejor calidad que reflejen una mayor individualidad y contribución con el medio ambiente.

En función de todas estas características y transportándolo al desarrollo de viviendas unifamiliares, las casas que cuentan con instalaciones que puedan albergar internet, telefonía, servicios de televisión por cable, aire acondicionado y ahorros de energía con calentadores solares y lámparas led que sean abastecidas por paneles solares; tuvieron una mayor aceptación entre las personas. Por tanto, se tuvo una mejor valoración y aumentan las preferencias por este tipo de inmuebles.

Las generaciones con mayor potencial para comprar una vivienda a partir del 2015 y hasta 2027, son los millennials (entre 1980 y 1995). La generación X (entre 1965 y 1979) que es la que les precede también son clientes potenciales, pero en menor porcentaje que los primeros. Estos eligen por estar a la vanguardia, tener diseños innovadores y nuevos que refleje a un hombre contemporáneo y moderno, por tal razón, se vuelve de vital importancia conocer sus gustos y estilo de vida. Para comunicarse, la generación X utiliza tanto el correo electrónico y el teléfono, como otras más innovadoras, tanto WhatsApp o chats en redes sociales. Los millennials son nativos digitales, no imaginan una vida sin el uso de tecnología y el internet para el entorno laboral y personal (Muticonversion, 2019).

Además, es importante mencionar que 6 de cada 10 créditos que otorgó el INFONAVIT en 2012, fueron otorgados a los nacidos entre 1983 y 2000 (Obras, 2018). Esto es un notificado que se atribuye al portal inmobiliario propiedades.com, así como, el $49 \%$ de las búsquedas para obtener una casa proviene de la generación " $Y$ " o milenialls, en segundo lugar, se encuentran quienes tienen de 35 a 44 años, con 26\%; y $12 \%$ de 45 a 54 años (NOTIMEX, 2018).

El impacto que tienen estos datos conlleva a dar mayor valor a las propiedades que cumplan con todos los requerimientos de las generaciones antes mencionadas, porque al 2020, son las que se encuentran en posibilidades económicas de adquirir una vivienda. Es 
ahí donde surge la necesidad de valorar un proyecto arquitectónico de manera integral, que pondere la funcionalidad, su forma, estética y contribución con el medio ambiente, además de contar con todos los servicios que permitan una vida confortable.

El objetivo de la investigación fue categorizar la valuación de viviendas unifamiliares como medio de control en Orizaba, Veracruz, México y para tal efecto se presenta la metodología siguiente.

\section{Metodología}

Para llevar a cabo la recopilación de la información del bien tangible a valuar que se refiere al tipo de viviendas en la ciudad de Orizaba, Veracruz, México, fue necesario realizar un estudio comparativo de mercado, en donde se investigó lo concerniente al estudio.

Para ello, se especificó la zona en donde se desarrollaron este tipo de proyectos y que contaron con un mayor equipamiento y servicios que otras partes de la ciudad. Posteriormente, se ubicó en el mapa la zona de estudio y se realizó un recorrido para ubicar las casas que tuvieran características similares y que pudieran ser equiparables entre sí, para dar mayor certeza al proyecto.

Una vez ubicadas las viviendas se estableció la casa tipo que fuera objeto de estudio, para saber si su precio de venta fue el correcto, de esta manera se podría determinar el valor unitario de mercado. Con esta vivienda se identificaron los elementos exógenos de su ubicación y se describieron los elementos endógenos del inmueble. Para ello, fue necesario solicitar permiso a un habitante de Residencial La Joya, quien accedió a brindar información acerca de su vivienda y realizar una visita de campo a su domicilio para recopilar los datos necesarios. Esta casa rigió la muestra de comparables en la zona de estudio.

Para recopilar los datos de las viviendas que sirvieron como comparables, se accedió a la página web donde las promovieron y se habló por teléfono al número que tenía publicado para dar informes; además, de acudir a la zona de estudio y visitar el lugar donde se encontraban. De igual manera, se realizará un estudio de mercado en zonas diversas de la ciudad, así se obtuvieron comparables de características similares al bien inmueble 
tomado como referencia. Estos datos fueron la base del avalúo para el enfoque comparativo de mercado.

Las estrategias utilizadas para la valoración fueron las siguientes:

- Realizar una planeación calendarizada de la ejecución del proyecto.

- Establecer recursos materiales, financieros y humanos para desarrollar el proyecto.

- Investigación de campo (reconocimiento de la zona donde se desarrollará el proyecto, toma de fotografías del bien inmueble, levantamiento de la infraestructura, servicios, actividad económica y equipamiento urbano).

- Estudio de mercado (investigación de comparables similares al bien que se va a valuar) en la zona de estudio.

- Análisis de la recopilación de datos.

- Aplicación de los métodos de valuación utilizados para un avalúo comercial.

- Utilización de análisis de precios unitarios, costos paramétricos y programa Opus para realizar el método de costos (CMIC, BIMSA y ANÁLISIS DE P.U. OPUS).

- Determinación del precio de venta de una vivienda con el enfoque de las inmobiliarias.

- Aplicación de encuestas a la población para conocer el nivel de satisfacción en relación con los precios de venta con el diseño y construcción de las viviendas.

- Análisis de las variables que aumentan la plusvalía en la ciudad de Orizaba, Veracruz.

- Evaluación de precios de viviendas en diferentes zonas de la ciudad.

- Conclusiones del proyecto.

La metodología que se aplicó para este proyecto de intervención en el tema de la valuación y evaluación de precios de viviendas unifamiliares fue el "método de comercialización" que se empleó como medida de control en el mercado inmobiliario de la ciudad de Orizaba, Veracruz, México, fue una investigación de tipo cuantitativo con alcances correlacionales, en el cual, se estudiaron las relaciones de las variables de la investigación. Para ello, fue importante medirlas, cuantificarlas, analizarlas y establecer las vinculaciones pertinentes que cumplieran con los objetivos del estudio. El número de inmuebles que se investigó en la zona de estudio fue de seis comparables, de acuerdo con la normatividad mexicana de valuación NMX-R-081-2015. 
Al término de cada método y después de obtener los valores de los diferentes enfoques (método comparativo de mercado, de costos y capitalización), se presentó un resumen de valores y el tipo de valor que es concluido, se obtiene en función al propósito del avalúo, señalándose en su caso, aquellos factores o condiciones particulares que hubieran influido, significativamente, en su determinación (DOF, 2006).

Por otro lado, la técnica de depreciación elegida para la valuación de bienes inmuebles por el método de costos o reproducción del bien es: MÉTODO DE LÍNEA RECTA.

Para llevar a cabo este método fue necesario asignar una vida útil al bien que se valoró. En el caso de bienes inmuebles el valuador determinó la vida útil de la edificación e investigó su fecha en que se construyó.

La fórmula para llevar a cabo la depreciación por este método es:

$$
D=(V R-V r) K
$$

Donde:

$\mathrm{Edad}=\mathrm{E}$

Vida útil $=$ Vu

Valor de Reposición (Nuevo) = VR

Valor de reposición $(10 \%$ del VR) $=\mathrm{Vr}$

Coeficiente que relaciona la antigüedad con la vida útil $=\mathrm{K}$

Este método supone que la depreciación anual del activo fijo es la misma durante cada año de su vida útil y, por consiguiente, se considera como un incremento progresivo de la depreciación. La depreciación calculada de esta manera, puede utilizarse para inmuebles que su estado de conservación se encuentra en condiciones normales y que no han sufrido remodelaciones o mantenimientos importantes.

Por consiguiente, y si se busca obtener el valor comercial de las viviendas que refleje su justo valor y no exceda del mismo provocando un alza de precios en los inmuebles, se optó por ponderar los dos valores que hayan estado más cerca el uno del otro (obteniendo el promedio), o en su defecto y si dista mucho entre los tres valores, se optó por concluir por el valor que esté al centro (punto medio = mediana) del menor y mayor valor. De tener 
resultados que no equidistan en demasía entre ellos, se pudo promediar entre los tres valores, obteniendo así el valor final del avalúo.

A continuación, se adjunta el instrumento de recolección de datos para la evaluación de diseño de inmuebles empleado en el estudio.

\section{Tabla 1. Instrumento de escala de evaluación de proyecto arquitectónico}

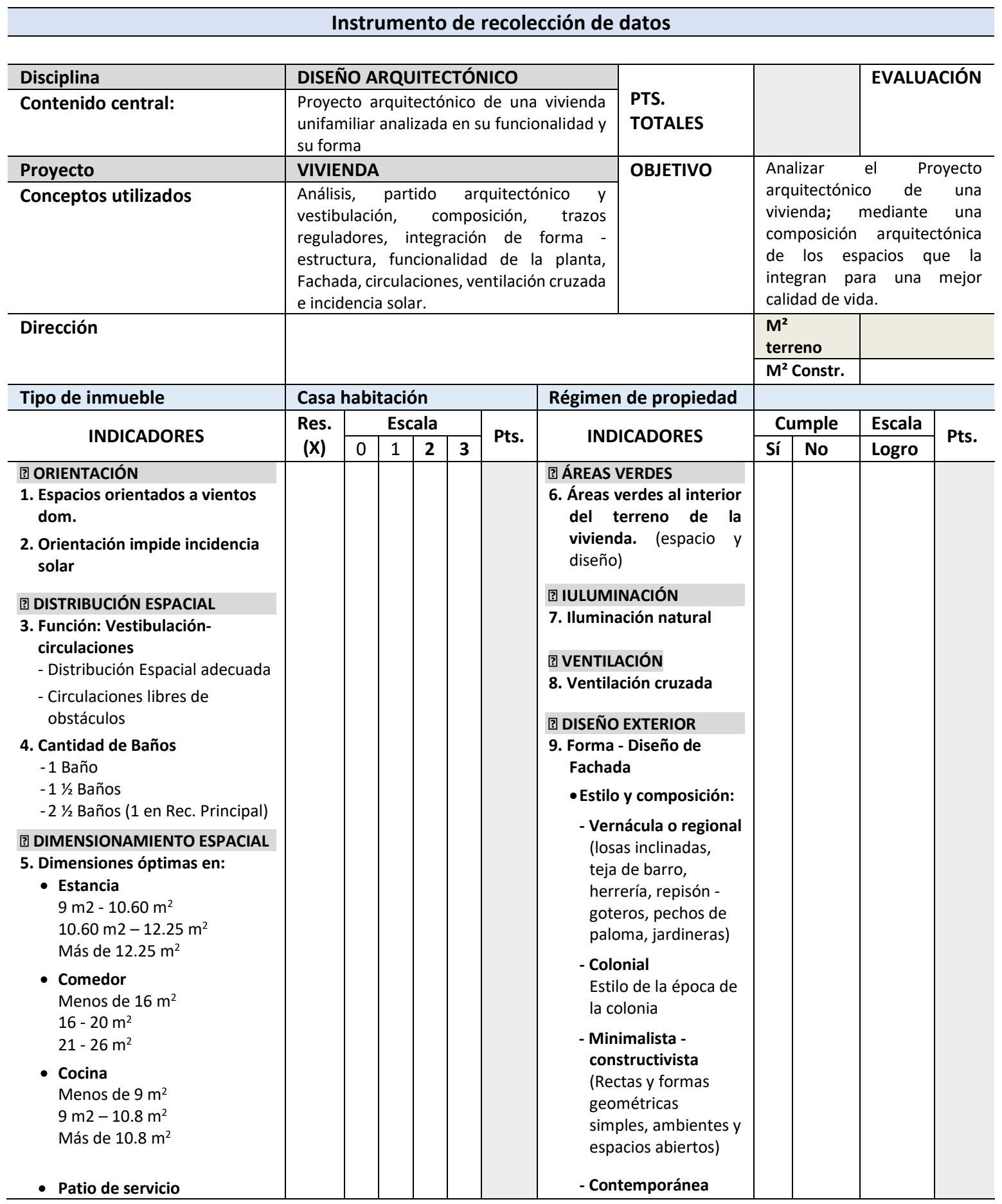




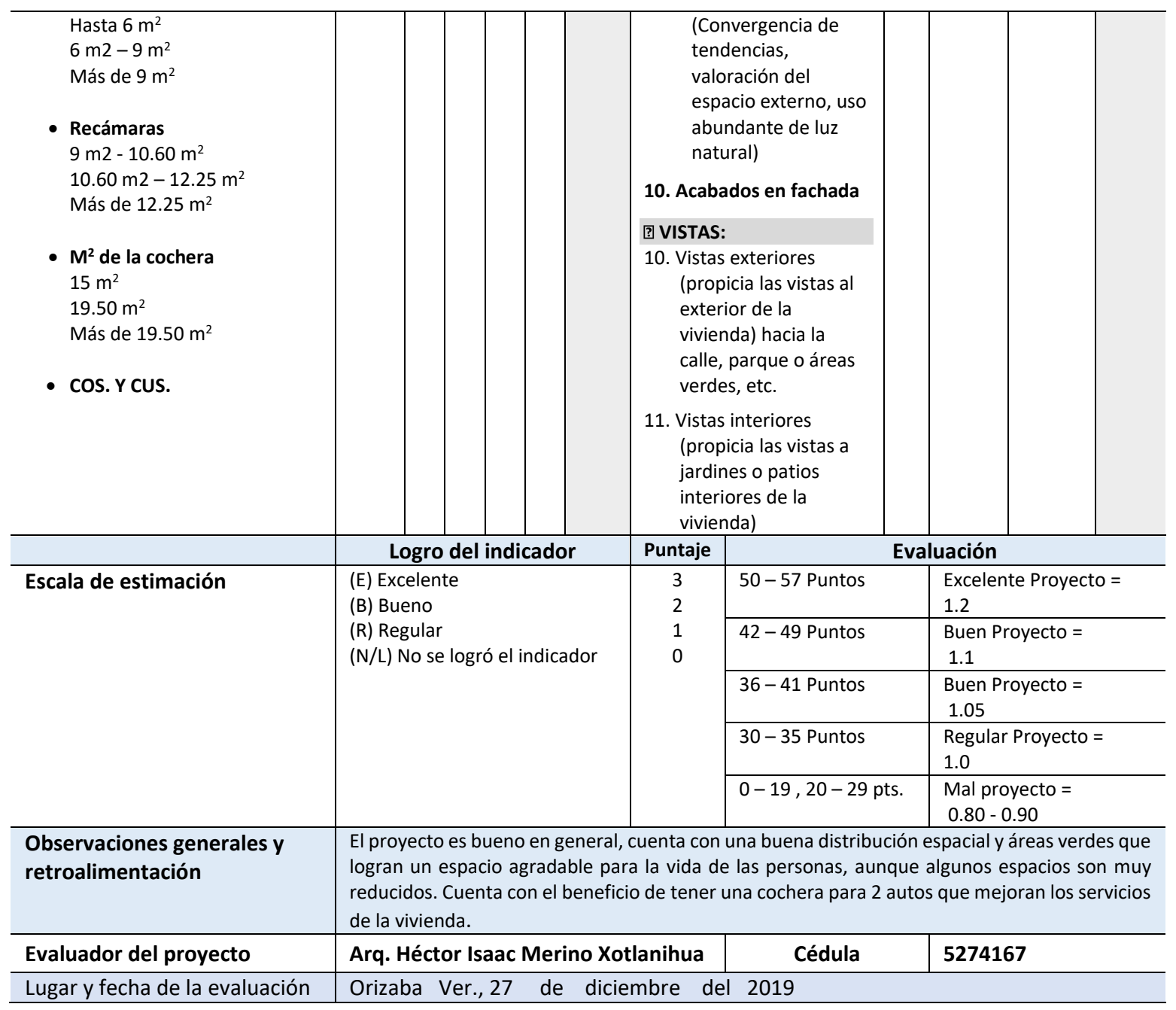

Fuente: Elaboración propia.

\section{Resultados y discusión}

Retomando el valor físico del avalúo inmobiliario se tiene que:

$\$ 1,269,940$ pesos mexicanos $(63,528$ dólares americanos) que ya contempla el factor resultante de la plusvalía del terreno de acuerdo con la zona y al equipamiento urbano con el que cuenta.

Cálculo del factor resultante de la calidad del proyecto arquitectónico.

Multiplicación del Valor Físico por el Factor resultante de la Calidad del Proyecto Arquitectónico:

$$
V F * \operatorname{Fr}(C P A)=\text { Valor del inmueble }
$$

$\$ 1,269,940.00 * 1.05=\$ 1,333,437.00$ 
Que siendo redondeado queda de $\$ 1,330,000$ pesos mexicanos $(66,533$ dólares americanos) como precio de venta del inmueble en el 2019, el cual, fue vendido en $\$ 1,380,000$ pesos mexicanos (69,034 dólares americanos) en enero de 2017. En diciembre de 2019 fueron vendidos en $\$ 1,475,000$ pesos mexicanos (73,786 dólares americanos) y $\$ 1,500,000$ pesos mexicanos $(75,037$ dólares americanos), el mismo proyecto arquitectónico con los mismos $\mathrm{m}^{2}$ de terreno y de construcción, pero insertado en otro fraccionamiento (Residencial Habitat) en diferente zona de la ciudad con características similares al residencial La Joya en donde se llevó a cabo el ejercicio de valuación de una vivienda.

Se observó que los precios por $\mathrm{m}^{2}$ que imperó en la zona, distan mucho uno de otro, por lo tanto, no se pudo tomar un dato exacto del valor de mercado, debido a que varios inmuebles estaban sobre valuados y no reflejaron su justo valor. No obstante, al aplicar los factores de homologación de comparables, el valor promedio estaba por encima del valor real del inmueble $\$ 11,109.00 / \mathrm{m}^{2}$ pesos mexicanos ( $\$ 555.72$ dólares americanos). El valor de acuerdo con estudio de mercado en Orizaba, ciudades circunvecinas importantes como fueron: el Puerto de Veracruz, Puebla y Córdoba, osciló entre un límite inferior de $\$ 9,900 / \mathrm{m}^{2}$ (\$495.24 dólares americanos) y un límite superior de $\$ 10,600 / \mathrm{m}^{2}$ pesos mexicanos (\$530.26 dólares americanos) (según la zona y equipamiento) para viviendas de interés medio entre 120 y $145 \mathrm{~m}^{2}$ de construcción y una superficie de terreno entre 105 y $135 \mathrm{~m}^{2}$.

Los resultados señalan, que sí existió un incremento en las viviendas de la ciudad de Orizaba en relación a ciudades con infraestructura y desarrollo económico mayor como fueron Veracruz y Puebla, las cuales, se encuentran a una hora y 20 minutos de la ciudad mencionada. Por otra parte, es importante decir, que la ciudad ha ido creciendo en equipamiento urbano que mejora los servicios de sus habitantes y en desarrollo turístico, que a su vez, contribuyen a una derrama económica importante; sin embargo, otras ciudades cuentan con esta misma característica y los precios de las viviendas son más bajos, por tal razón, existe un incremento notorio en Orizaba que provoca un impacto económico, 
no sólo en la adquisición de vivienda, si no, en el pago de impuestos y en el comercio al adquirir cualquier otro producto o servicio. Ver tabla 2 y 3.

Tabla 2. Comparativa de precios

\begin{tabular}{|c|c|c|c|c|c|c|c|c|}
\hline \multirow{3}{*}{ Inmueble } & \multicolumn{2}{|c|}{ Orizaba - ene/2019 } & \multirow{2}{*}{$\frac{\text { IMIC-CMIC }}{\text { tabla }}$} & \multirow{2}{*}{$\begin{array}{l}\text { Veracruz } \\
\text { dic. } 2019\end{array}$} & \multirow{2}{*}{$\begin{array}{l}\text { Puebla } \\
\text { dic. } 2019\end{array}$} & \multicolumn{3}{|c|}{ Diferencias con Orizaba } \\
\hline & $\begin{array}{c}\text { tabla } 3 \\
\text { investigación } \\
\text { de campo }\end{array}$ & $\begin{array}{c}\text { tabla } 4 \\
\text { investigación } \\
\text { de campo }\end{array}$ & & & & IMIC-CMIC & Veracruz & Puebla \\
\hline & $\begin{array}{c}\text { precio / m² } \\
\text { zona-estudio }\end{array}$ & $\begin{array}{c}\text { precio } / \mathrm{m}^{2} \\
\text { general en la } \\
\text { ciudad }\end{array}$ & precio / $\mathrm{m}^{2}$ & precio $/ \mathrm{m}^{2}$ & precio $/ \mathrm{m}^{2}$ & $\begin{array}{c}\text { incremento + } \\
\text { decremento - } \\
\%\end{array}$ & $\begin{array}{c}\text { incremento + } \\
\text { decremento - } \\
\%\end{array}$ & $\begin{array}{c}\text { incremento }+ \\
\text { decremento - } \\
\%\end{array}$ \\
\hline \multirow{2}{*}{$\begin{array}{c}\text { Vivienda } \\
\text { unifamiliar } \\
\text { interés } \\
\text { medio }\end{array}$} & $\$ 11,169.00$ & $\$ 10,951.45$ & \multirow[b]{2}{*}{$\$ 10,533.00$} & \multirow[b]{2}{*}{$\$ 10,827.28$} & \multirow[b]{2}{*}{$\$ 11,084.00$} & \multirow[b]{2}{*}{$+5.4 \%$} & \multirow[b]{2}{*}{$+2.46 \%$} & \multirow[b]{2}{*}{$0 \%$} \\
\hline & $\begin{array}{r}\text { Prom } \\
\$ 11,060.23=\end{array}$ & $\begin{array}{l}\text { edio: } \\
\qquad 11,100.00\end{array}$ & & & & & & \\
\hline
\end{tabular}

Fuente: Elaboración propia.

Tabla 3. Comparativa de precios

\begin{tabular}{|c|c|c|c|c|c|c|c|c|}
\hline \multirow{3}{*}{ Inmueble } & \multicolumn{2}{|c|}{ Orizaba - sep/2019 } & \multirow{3}{*}{$\begin{array}{c}\text { IMIC- } \\
\text { CMIC } \\
\text { precio / m² }\end{array}$} & \multirow{3}{*}{$\begin{array}{l}\text { Veracruz } \\
\text { precio / m² }\end{array}$} & \multirow{3}{*}{$\begin{array}{c}\text { Puebla } \\
\text { precio / m² }\end{array}$} & \multicolumn{3}{|c|}{ Diferencias con Orizaba } \\
\hline & $\begin{array}{c}\text { tabla } 5 \\
\text { ventas } \\
\text { FOVISSSTE }\end{array}$ & $\begin{array}{c}\text { tabla } 6 \\
\text { ventas } \\
\text { FOVISSSTE }\end{array}$ & & & & IMIC-CMIC & Veracruz & Puebla \\
\hline & $\begin{array}{l}\text { precio } / \mathrm{m}^{2} \\
\text { en la ciudad }\end{array}$ & $\begin{array}{c}\text { precio / } \mathrm{m}^{2} \\
\text { zona- } \\
\text { estudio }\end{array}$ & & & & $\begin{array}{c}\text { incremento }+ \\
\text { decremento - } \\
\%\end{array}$ & $\begin{array}{c}\text { incremento }+ \\
\text { decremento - } \\
\%\end{array}$ & $\begin{array}{c}\text { incremento } \\
\text { decremento - } \\
\%\end{array}$ \\
\hline \multirow{2}{*}{$\begin{array}{c}\text { Vivienda } \\
\text { unifamilia } \\
r \text { interés } \\
\text { medio }\end{array}$} & $\$ 12,210.24$ & $\$ 12,513.76$ & \multirow[b]{2}{*}{$\$ 10,533.00$} & \multirow[b]{2}{*}{$\$ 10,827.28$} & \multirow[b]{2}{*}{$\$ 11,084.00$} & \multirow[b]{2}{*}{$+17.73 \%$} & \multirow[b]{2}{*}{$+12.68 \%$} & \multirow[b]{2}{*}{$10.62 \%$} \\
\hline & $\begin{array}{r}\text { pror } \\
\$ 12,362.00\end{array}$ & $\begin{array}{l}\text { dio: } \\
\$ 12,400.00\end{array}$ & & & & & & \\
\hline
\end{tabular}

Fuente: Elaboración propia

\section{Conclusiones}

- Las observaciones de este trabajo no es condicionar el mercado inmobiliario ni de atentar contra las utilidades de las empresas constructoras e inmobiliarias, sino, recibir el pago justo por el trabajo devengado y mantener una economía que sea benéfica para la población. Se considera que los incrementos deben ir en función de los Índices Nacionales de Precios al Productor (INPP) y de los Índices Nacionales de Precios al Consumidor (INPC), así como en función de la evaluación del proyecto arquitectónico, sus acabados y de la zona en donde se han construido para dar un precio de venta; que, de acuerdo con el poder adquisitivo de las familias, estas puedan elegir y adquirir una vivienda digna según sus posibilidades económicas. 
- Para poder emitir las conclusiones de este proyecto, fue necesario realizar un avalúo formal basado en la normatividad de la Sociedad Hipotecaria Federal, en donde se establece que, para determinar el valor comercial de un inmueble, es necesario realizarlo mediante tres diferentes enfoques de valuación como son: Enfoque de mercado, de costos y de ingresos.

- La finalidad del avalúo realizado fue determinar el valor de la vivienda y compararlo con el precio al que fue vendida, para verificar si existió un incremento injustificado en su valor.

- También se realizaron comparativas entre el resultado del avalúo y el precio de venta de algunas viviendas (con características similares) que se encuentren en la ciudad, así como, las que se ubican en otras ciudades de infraestructura y equipamiento mayor, para constatar si no existió un alza de precios en relación con las casas que se ofertan en Orizaba, Veracruz.

- Posterior al avalúo se propuso un método híbrido en el que se retoma el valor físico de la vivienda (enfoque de costos), al que se le adiciona la evaluación del proyecto arquitectónico para incrementar o demeritar su valor comercial.

- El comparativo de precio de los materiales, reflejó que las construcciones de vivienda, son $29 \%$ más costosas en el puerto de Veracruz que en la ciudad de Orizaba; sin embargo, se ofertan y venden a mayor precio las construidas en Orizaba. Lo anterior es producto de que Orizaba es una ciudad pequeña con poca extensión territorial (27.97 $\mathrm{km}^{2}$ ) y escasez de fracciones de suelo disponible para la construcción de vivienda unifamiliar. De 2014 al 2019 los terrenos más grandes de la ciudad fueron comprados por desarrolladoras inmobiliarias que se dedicaban a la producción de vivienda en serie o a fraccionar los terrenos a los que se les dotaba de infraestructura y servicios públicos para después ser vendidos y que cada persona desarrollará su vivienda.

- Los insumos con los que, generalmente, se construyen las viviendas tienen menor sobre costo en Orizaba que en otras ciudades. Este es un indicador (de varios que existen) de que el mercado inmobiliario está sobrevaluado al momento de asignarle el precio de venta a las casas que serán comercializadas. 
- Se determinó que, en la ciudad de Orizaba, Veracruz, los inmuebles se encuentran sobrevalorados y no refleja un precio justo de acuerdo con su nivel de equipamiento, infraestructura y mobiliario, por otro lado, se señala que el método de comercialización propuesto en esta investigación resulta de utilidad para los valuadores de la zona de estudio y pudiera replicarse y extrapolar a otros lugares, tomando en cuenta todas las condiciones de valoración señaladas.

\section{Referencias bibliográficas}

Carrillo, C. (29 de Octubre de 2018). Se dispara plusvalía en Orizaba. El mundo de Orizaba (17725),

Recuperado

de https://www.diarioelmundo.com.mx/index.php/2018/10/29/casas-de-orizabadelas-mas-caras/

Cuadernilos municipales, 2. G. (21 de 05 de 2016). CEIEG Veracruz - Gobierno de Veracruz. Recuperado de sistema de información municipal: http://ceieg.veracruz.gob.mx/wpcontent/uploads/sites/21/2016/05/Orizaba.pdf

DOF. (3 de Marzo de 2006). Anexo 42. Lineamientos generales para la valuación bancaria. DOF, resolución primera, 31. Recuperado de https://www.abm.org.mx/legislacion-yregulacion/juridico/anexoscub/CUB_Anexo42.pdf

Gonzales, P., Turmo, J., y Villaronga, E. (2006). La valoración inmobiliaria teoría y práctica. España: Ediciones Gráficas Muriel SA.

INDAABIN. (20 de Marzo de 2019). Marco normativo en materia valuatoria. (S. G. México, Editor). Recuperado de glosario de términos en materia de avalúos. Dirección General de Avalúos y Obras: https://www.gob.mx/cms/uploads/attachment/file/446970/Glosario de Terminos $20 \quad 03$ 2019.pdf

López, A., Aznar, J., Guijarro, F., y González, R. (2012). Valoración inmobiliaria. Métodos y aplicaciones (España e Iberoamérica).

Meca, E., Conesa, I., y Briones, J. (2009). Normas internacionales de valoración: la opinión de los profesionales en España. 
Muticonversion. (27 de Mayo de 2019). Multiconversion Roi Agency. Recuperado de https://multiconversion.com/millennials-generacion-z-generaciones-marketing/

Moctezuma, M., y Veyna, O. (2006). Remesas colectivas, estado y formas organizativas de los mexicanos en EUA. El Programa $3 \times 1$ para migrantes: Primera política transnacional en México, 119-138.

NOtIMEX, A. (7 de Agosto de 2018). Milenio 2020. Recuperado de https://www.milenio.com/negocios/millennials-si-quieren-invertir-en-unapropiedad

Ochoa, J. (10-14 de Noviembre de 2017). México desconocido.com. (J. Ochoa, Editor) Recuperado de https://www.mexicodesconocido.com.mx/orizaba-pueblo-magicofavorito-los-viajeros.html

SHCP, S. d. (9 de Febrero de 2018). Índice nacional de precios al consumidor 2018. Recuperado de http://www.sat.gob.mx/informacion fiscal/tablas indicadores/Paginas/inpc 2018.a $\underline{\mathrm{spx}}$ 